\title{
Investigation of the recombination of the retarded shell of "born-again" CSPNe by time-dependent radiative transfer models
}

\author{
Antti Koskela ${ }^{1}$, Silvia Dalnodar ${ }^{2}$, Ralf Kissmann ${ }^{2}$, Anita Reimer ${ }^{3}$, \\ Alexander Ostermann ${ }^{1}$ and Stefan Kimeswenger ${ }^{2}$ \\ ${ }^{1}$ Department of Mathematics, University Innsbruck, Technikerstrasse 13/7, \\ A-6020 Innsbruck, Austria \\ ${ }^{2}$ Institute of Astro- and Particle Physics, University Innsbruck, Technikerstrasse 25/8, \\ A-6020 Innsbruck, Austria \\ ${ }^{3}$ Institute of Theoretical Physics, University Innsbruck, Technikerstrasse 25/2, \\ A-6020 Innsbruck, Austria
}

\begin{abstract}
A standard planetary nebula stays more than 10000 years in the state of a photoionized nebula. As long as the timescales of the most important ionizing processes are much smaller, the ionization state can be characterized by a static photoionization model and simulated with codes like CLOUDY (Ferland et al. 1998). When the star exhibits a late helium flash, however, its ionizing flux stops within a very short period. The star then re-appears from its opaque shell after a few years (or centuries) as a cold giant star without any hard ionizing photons. Describing the physics of such behavior requires a fully time-dependent radiative transfer model. Pollacco (1999), Kerber et al. (1999) and Lechner \& Kimeswenger (2004) used data of the old nebulae around V605 Aql and V4334 Sgr to derive a model of the pre-outburst state of the CSPN in a static model. Their argument was the long recombination time scale for such thin media. With regard to these models Schönberner (2008) critically raised the question whether a significant change in the ionization state (and thus the spectrum) has to be expected after a time of up to 80 years, and whether static models are applicable at all.
\end{abstract}

Keywords. radiation mechanisms: general, ISM: general, planetary nebulae: individual (V604 Aql, V4334 Sgr)

We use the transport equation formalism presented in the appendix of Binette et al. (2003) to describe the evolution of the nebula after the rapid change of the central star. As extensions to their model we also take He and the CNO elements into account, both as source of free electrons and as absorbing species. Also, we use a realistic stellar photosphere model for the input radiation field, and, as we investigate the evolution for a large change of the input radiation field, we do not use their monochromatic approach of a single mean frequency. The system is solved using a classical splitting scheme and the pressure and temperature are calculated using an explicit solver. The physical parameters were taken from Osterbrock \& Ferland (2006) and Gnat \& Sternberg (2007). The initial conditions were calculated by CLOUDY. For the evolution of the "born-again" star in the Hertzsprung-Russell-Diagramm the tracks as used in van Hoof et al. (2007) were applied.

The nebulae of the "born-again" central stars recombine extremely slowly. The hydrogen and helium timescales dominate the whole process. Pollacco (1999) raised the question about the oxygen recombination time scale. As single species it would be only about one decade. The coupling of the oxygen processes with that of the helium, however, slows down the recombination, as long as He II exists, to a rate even below that of hydrogen. 


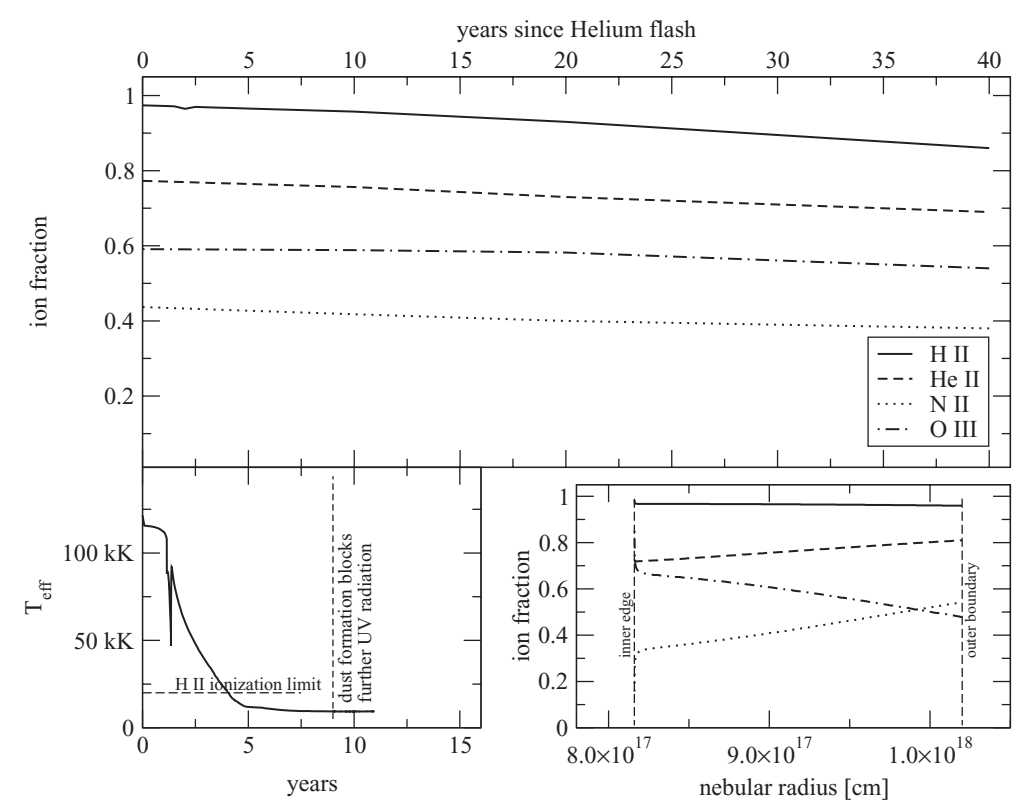

Figure 1. The upper panel shows the resulting time evolution of the ionization state of the nebula. We show only those ions which were used for photoionization analysis for the pre-flash white dwarfs. The state at the mean radius of the nebula is shown here. The solution of a model including the cooling luminous star while returning to the AGB (lower left panel), and that completely without any ionizing source, differ by less than the line thickness. The lower right panel shows the spatial distribution after 20 years. Due to the low filling factor, the time evolution turns out to be similar for all radii. In contrast to the simulation by Binette et al. (2003), no outer recombination rim is formed.

The ionization of the luminous, but much colder star during the transit back to the AGB phase, stated by Schönberner (2008) as possible major mechanism for reionization, is negligibly small. Thus the previous results of the pre-flash state of V4224 Sgr (Pollacco 1999 and Kerber et al. 1999) and V605 Aql (Lechner \& Kimeswenger 2004) are reliable.

\section{Acknowledgements}

A.K. and S.D. are funded by Austrian Science Fund (FWF) DK+ project Computational Interdisciplinary Modeling, W1227.

\section{References}

Binette, L., Ferruit, P., Steffen, W., \& Raga, A. C. 2003, Rev. Mexicana AyA, 39, 55

Ferland, G. J., Korista, K. T., Verner, D. A., Ferguson, J. W., Kingdon, J. B., \& Verner, E. M. 1998, PASP, 110, 761

Gnat, O. \& Sternberg, A. 2007, ApJS, 168, 213

Kerber, F., Köppen, J., Roth, M., \& Trager, S. C. 1999, A\& A, 344, L79

Lechner, M. F. M. \& Kimeswenger, S. 2004, A\& A, 426, 145

Osterbrock, D. E. \& Ferland, G. J. 2006, Astrophysics of gaseous nebulae and active galactic nuclei, Sausalito, CA: University Science Books

Pollacco, D. 1999, MNRAS, 304, 127

Schönberner, D. 2008, in: K. Werner \& T. Rauch (eds.) Hydrogen-Deficient Stars, Proc. ASP Conference Series, Vol. 391 (San Francisco: ASP), p. 139

van Hoof, P. A. M., Hajduk, M., Zijlstra, A. A., Herwig, F., Evans, A., van de Steene, G. C., Kimeswenger, S., Kerber, F., \& Eyres, S. P. S. 2007, A\& $A$ 471, L9 